\title{
Projection of non-singular projective varieties
}

\author{
By \\ Audun Holme \\ (Communicated by Professor Nagata, March 6, 1972) \\ (Revised, October 12, 1972)
}

\section{Introduction}

It is a classical result (cf. [3]) that a non-singular projective variety $X \hookrightarrow \mathbf{P}_{k}^{N}$ over an algebraically closed field $k$ may be embedded in $\mathbf{P}_{k}^{2 n+1}, n=\operatorname{dim}(X)$, via a projection from $\mathbf{P}_{k}^{N}($ if $N>2 n+1)$. There are examples which show that in general this can not be done for $\mathbf{P}_{k}^{2 n}$.

The second best to an embedding induced by a projection is the following: A birational model $Y$ of $X$ in $\mathbf{P}_{k}^{2 n}$, together with a birational morphism $\lambda: X \rightarrow Y$ induced by a projection, and such that $Y$ differs from $X$ by as little as possible.

So the question is: How well can one do with this $Y$ ?

For curves the answer to this problem is again classical. The singularities of $Y$ are at most ordinary double points, and $\lambda$ is an isomorphism at all regular points of $Y$ (cf. Chapter I, $\S 1$ for the meaning of "isomorphism at a point").

In higher dimensions the problem has been considered by E. Lluis [4], J. Roberts [7], and the author [2].

In [4], Lluis proves that $Y$ has at most a finite number of singularities, which have linear analytic branches, i.e., Spec of the completions of the local rings at the singular points are fimite unions of regular schemes.

In [7], Roberts shows that if the embedding of $X$ into $\mathbf{P}_{k}^{N}$ is sufficiently nice (and all projective varieties can be given such nice 
projective embeddings) then $Y$ has at most a finite number of double points, with linear analytic branches - and moreover, these have normal crossings. This last statement is equivalent to that the tangent cone $\bar{C}_{Y, y}$ (cf. Chapter II, §2) has two components each of which are a $\mathbf{P}^{n}$, in general position to each other, at all singular points $y$ of $Y$.

The author has obtained, (cf. [2]) as a consequence of a certain formal projection theorem, that without any condition on the embedding of $X, Y$ has at most double points. But a priori the tangent spaces of the analytic branches at the double point $y \in Y$ might coincide, i.e. $\bar{C}_{Y, y}$ might have only one irreducible component (and as a scheme $\bar{C}_{Y, y}$ would then necessarily be non-reduced). According to Roberts' theorem above this could, of course, only occur if the embedding of $X$ into $\mathbf{P}_{\boldsymbol{k}}^{N}$ were not nice in his sense.

For curves we have already mentioned that this does not happen. The purpose of the present work is to extend that result to higher dimensions.

We first list some well known facts and establish the notation in Chapter I. Most of this material is more or less readily available in the literature.

The reader could go directly to Chapter II, and refer back when needed.

Chapter II opens with the study of projective embeddings $X \hookrightarrow \mathbf{P}_{k}^{N}$ $N=2 \operatorname{dim}(X)+1$, with the property that for any two non-singular $k$ points $x$ and $y$ of $X$, the tangent spaces $T_{X, x}$ and $T_{X, y}$ span a space $<T_{X, x}, T_{X, y}>$ of dimension $\leq n+1, n=\operatorname{dim}(X)$. Such subvarieties are characterized in Theorem 1.1. This theorem implies that if $X$ above is non-singular, then it must be contained in a hyperplane in $\mathbf{P}_{k}^{N}$, Theorem 1.3. Using this information we finally show the result announced above, namely Theorem 3.1 .

Even if $\bar{C}_{Y, y}$ has two distinct components at all the double points of $Y$, those components naturally need not be in general position, as in Roberts theorem. The question of whether this remains true without any condition on the embedding $X \hookrightarrow \mathbf{P}_{k}^{N}$ is still open. The essential point here is to obtain a classification, analogous to Theorem 1.1, of 
those $X \hookrightarrow \mathbf{P}_{k}^{N}$ which satisfy

$$
\operatorname{dim}<T_{X, x}, T_{X, y}>\leq 2 n-1 \quad(n=\operatorname{dim}(X))
$$

for all non-singular $x, y$ in $X$.

Unfortunately, the rather simple techniques used in the proof of Theorem 1.1 do not seem to be sufficient for this. However, recently the author has obtained some results which indicate that a different approach could settle it:

Namely, if $P$ denotes the blowing up of $\mathbf{P}_{k}^{N} \times{ }_{k} \mathbf{P}_{k}^{N}$ with center in the diagonal, one can define certain generators $t, \xi$ and $\zeta$ for the Chow ring $A(P)$. We shall not go into details here, only say that $t^{N+1}=0, \xi$ satisfies an equation of degree $N$ with coefficients from $Z[t]$, and $\zeta$ one of degree 2 with coefficients from $Z[t, \xi]$.

Now let $\varphi: X \hookrightarrow \mathbf{P}_{k}^{N}$ be the closed embedding of a non-singular subvariety (as before $N=2 \operatorname{dim}(X)+1$ ), and let $\widetilde{X \times{ }_{k} X}$ be the strict transform of $X \times{ }_{k} X$ in $P$. Then

$$
c l\left(\widehat{X \times{ }_{k} X}\right)=\left(\sum_{i=1}^{N} a_{i}(\varphi) t^{i} \xi^{N-i}\right) \zeta+\sum_{j=0}^{N+1} b_{j}(\varphi) t^{N+1-j} \xi^{j}
$$

We can show that $X$ may be embedded into $\mathbf{P}_{k}^{N-1}$ via a projection from $\mathbf{P}_{k}^{N}$ if and only if

$$
\sum_{i=1}^{N}(-1)^{i} a_{i}(f)=0 .
$$

Now the nature of the generators above seems to indicate that (*) may be translated into certain algebraic relations among the $a$ 's and $b$ 's above. If this can be done, then the next question is whether those relations imply (**). If so, Roberts theorem follows without any conditions on the embedding $\varphi$. On the other hand, if there exists $\varphi: X \hookrightarrow \mathbf{P}_{k}^{N}$ such that the relations hold for the $a$ 's and $b$ 's, but (**) does not, then it gives a counterexample.

I would like to thank the referee for several improvements in the presentation of this material.

\section{Chapter I. Preliminaries}

§1. Basic definitions and notation. Throughout we use the nota- 
tion of EGA. In particular, if $\mathcal{O}$ is a local ring, $\mathfrak{m}_{\mathfrak{o}}$ denotes the maximal ideal. If $x$ is a point of the scheme $X$, then $\mathcal{O}_{X, x}$ and $\mathfrak{m}_{X, x}$ denote the local ring of $X$ at $x$, and its maximal ideal, respectively. If $X=\operatorname{Spec}(R), \mathfrak{p}(x)$ denotes the prime ideal of $R$ which corresponds to $x$, and $k(x)$ denotes the field $\mathcal{O}_{X, x} / \mathfrak{m}_{X, x}$.

Let $k$ be a field, which we always assume algebraically closed. Put $\mathbf{P}_{k}^{N}=\operatorname{Proj}\left(k\left[X_{0}, \ldots, X_{N}\right]\right)$. An $s$ dimensional linear subspace will be denoted by $P^{s}$. If $X$ and $Y$ are closed subschemes of $\mathbf{P}_{k}^{N}, X \cap Y$ and $X \cup Y$ denote the scheme-theoretic intersection and union, respectively: $X \cap Y$ is given by the sum of the homogeneous ideals $I(X)$ and $I(Y)$, and $X \cup Y$ is given by $I(X) \cap I(Y)$.

Further, if $X$ and $Y$ are projective schemes over $k, f: Y \rightarrow X$ a proper morphism and $Z$ a subscheme of $Y$, then $f(Z)$ denotes the closed scheme-theoretic image of $Z$, EGA I, 9.5. In particular the closure $\bar{Z}$ of $Z$ in $Y$ is the closed image by the canonical embedding. We say that $f$ is an isomorphism at $x \in X$ if $x$ is contained in an open subset $U$ such that the induced $f^{-1}(U) \rightarrow U$ is an isomorphism. The (closed) set of all points where $f$ is not an isomorphism is denoted by $B(f)$.

In some situations the following notation will be convenient. Let $S=k\left[Z_{i} \bar{Z}_{j} \mid 0 \leq i, j \leq N\right]$ be the subring of $k\left[Z_{0}, \ldots, Z_{N}, \bar{Z}_{0}, \ldots, \bar{Z}_{N}\right]$ generated over $k$ by the monomials $Z_{0} \bar{Z}_{0}, Z_{0} \bar{Z}_{1}, \ldots, Z_{N} \bar{Z}_{N} . S$ is graded by $\operatorname{deg}\left(Z_{i} \bar{Z}_{j}\right)=1$. Then $\operatorname{Proj}(S)=\mathbf{P}_{k}^{N} \times{ }_{k} \mathbf{P}_{k}^{N}$. Such notation is convenient for describing products of grassmanians.

Let $P=\left(p_{0}: \ldots: p_{N}\right)$ be a $k$-point of $\mathbf{P}_{k}^{N}=\operatorname{Proj}\left(k\left[X_{0}, \ldots, X_{N}\right]\right)$, where $p_{0} \neq 0$. Let $\pi_{P}: \widetilde{\mathbf{P}}_{k}^{N} \rightarrow \mathbf{P}_{k}^{N}$ be the blowing up with center $P$. We have the diagram

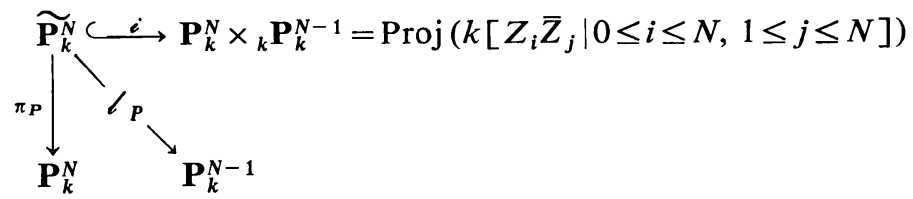

where $\pi_{P}$ is induced by $p r_{1}, \iota_{P}$ is induced by $p r_{2}$ and $i$ is the closed embedding which identifies $\mathbf{P}_{k}^{N}$ with the closed subscheme defined by 


$$
\begin{gathered}
Z_{i} \bar{Z}_{j}-Z_{j} \bar{Z}_{i}=0,1 \leq i, j \leq N . \\
\mathbf{P}_{k}^{N}=\operatorname{Proj}\left(k\left[X_{0}, \ldots, X_{N}\right]\right)=\operatorname{Proj}\left(k\left[Z_{0}, \ldots, Z_{N}\right]\right), \text { where } \\
Z_{0}=X_{0} \\
Z_{i}=p_{0} X_{i}-p_{i} X_{0}, \quad i=1, \ldots, N .
\end{gathered}
$$

Moreover, $\ell_{P}$ is a $\mathbf{P}^{1}$-bundle, and

$$
y \longmapsto \pi_{P}\left(/ \bar{P}^{-1}(y)\right)
$$

sets up a 1-1 correspondence between $k$-points of $\mathbf{P}_{k}^{N-1}$ and $P^{1}$ 's in $\mathbf{P}_{k}^{N}$ passing through $P$.

The morphism $\ell_{P}$ induces the projection

$$
\bar{\ell}_{P}: \mathbf{P}_{k}^{N}-\{P\} \rightarrow \mathbf{P}_{k}^{N-1} .
$$

If $X$ is a closed subscheme of $\mathbf{P}_{k}^{N}, \tilde{X}$ denotes the strict transform of $X$ by $\pi_{P}$, i.e., $\tilde{X}$ is the closure in $\widetilde{\mathbf{P}_{k}^{N}}$ of $\pi_{P}^{-1}(X-\{P\})$. This gives the commutative diagram

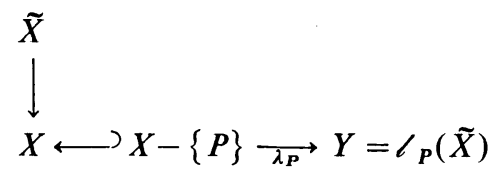

Let $G_{k}(N, r)$ denote the grassmanian which parametrizes $P^{r}$ 's in $\mathbf{P}_{k}^{N}$. Recall that for a fixed $P^{s}$ in $\mathbf{P}_{k}^{N}$, the set of points in $G_{k}(N, r)$ which correspond to $P^{r}$ 's containing $P^{s}$, form a closed subvariety which we denote by $\Delta\left(P^{s}\right)$. Furthermore, recall that

$$
G_{k}(N, r)=\operatorname{Proj}\left(k\left[T_{i_{0}, \ldots, i_{r}} \mid 0 \leq i_{\alpha} \leq N\right] / I\right),
$$

where the $T$ 's are indeterminates, and $I$ is a certain homogeneous ideal, generated by the Plücker relations. Put $t_{i}=T_{i} \bmod I, i=\left(i_{0}, \ldots\right.$, $\left.i_{r}\right)$. If $P^{r}$ is spanned by the points

$$
\left(a_{0,0}: \ldots: a_{0, N}\right), \ldots,\left(a_{r, 0}: \ldots: a_{r, N}\right),
$$

then the corresponding point of $G_{k}(N, r)$ has homogeneous coordinates $\left\{p_{i_{0}, \ldots, i_{r}}\right\}$ where $p_{i_{0}, \ldots, i_{r}}$ is the minor obtained from the $i_{0}, \ldots, i_{r}$ th. 
column of the matrix

$$
\left[\begin{array}{l}
a_{0,0}, \ldots, a_{0, N} \\
\ldots \\
a_{r, 0}, \ldots, a_{r, N}
\end{array}\right]
$$

It is easily seen from this that

$$
\operatorname{dim} G_{k}(N, r)=(r+1)(N-r)
$$

and

$$
\Delta\left(P^{s}\right) \approx G_{k}(N-s-1, r-s-1) .
$$

With the notation introduced above, write $\mathbf{P}_{k}^{N} \times{ }_{k} G_{k}(N, 1)=$ $\operatorname{Proj}\left(k\left[Z_{j} t_{i} \mid i=\left(i_{0}, i_{1}\right), 0 \leq i_{\alpha}, j \leq N\right]\right), \quad \quad \quad \mathbf{P}_{k}^{N} \times{ }_{k} \mathbf{P}_{k}^{N} \times{ }_{k} G_{k}(N, 1)=$ $\operatorname{Proj}\left(k\left[Z_{j_{1}} \bar{Z}_{j_{2}} t_{i} \mid i=\left(i_{0}, i_{1}\right), 0 \leq i_{\alpha}, j_{\beta} \leq N\right]\right)$. Let $\Gamma_{1}$ be the closed subscheme of $\mathbf{P}_{k}^{N} \times{ }_{k} G_{k}(N, 1)$ defined by the ideal generated by

$$
\sum_{\delta=0}^{2}(-1)^{\delta} Z_{i_{\delta}} t_{i_{0}, \ldots, \hat{i}_{\delta}, \ldots, i_{2}}
$$

where $\hat{i}_{\delta}$ means " $i_{\delta}$ omitted". $\Gamma_{1}$ so defined has the following property:

$$
\Gamma_{1}(k)=\{(x, \ell) \mid x \in \ell\},
$$

where $/$ denotes both a point of $G_{k}(N, 1)$ and the corresponding line.

Similarly one defines a closed subset of the second product above, $\Gamma_{2}$, for which $\Gamma_{2}(k)=\{(x, y, \zeta) \mid x, y \in \ell\}$.

The proof of the following proposition is quite straightforward:

Proposition 1.1.. If $T$ is a subscheme of $G_{k}(N, 1)$, then $p r_{1}\left(p r_{2}^{-1}(T) \cap \Gamma_{1}\right) \subset \mathbf{P}_{k}^{N}$ is the closure of the union of all lines in $\mathbf{P}_{k}^{N}$ which correspond to points from $T$.

Corollary 1.2. Let $S \subset \mathbf{P}_{k}^{N} \times{ }_{k} \mathbf{P}_{k}^{N}-\Delta_{P_{k}^{N} / k}$ be a subscheme of dimension $n$. For any $k$-points $p \in S$, denote by $L(p)$ the line passing through the points $\operatorname{pr}_{i}(p), i=1,2$. Let $L(S)$ be the closure of $\cup_{p \in S} L(p)$.

Then $\operatorname{dim} L(S) \leq n+1$. 
Proof. Denote the morphisms induced by the projections by<smiles>[3H][13CH3]</smiles><smiles>N#[Y10][Y10]#[In]</smiles>

Let $T=p_{3}\left(p_{12}^{-1}(S)\right)$. Then $L(S)=q_{1}\left(q_{2}^{-1}(T)\right)$ by the proposition. Hence it suffices to show that $\operatorname{dim} q_{2}^{-1}(T) \leq n+1$. As $q_{2}$ is a $\mathbf{P}^{1}$-bundle ([1], Proposition 1.3), this will follow if $\operatorname{dim} T \leq n$. But since $S \cap \Delta_{P_{k}^{N} / k}=$ $\emptyset, \operatorname{dim} p_{12}^{-1}(S) \leq n$, and the proof is complete.

The following proposition is well known and easy to prove. ${ }^{1)}$

Proposition 1.3 Let $X$ be an n-dimensonal subvariety of $\mathbf{P}_{k}^{N}$. Then there exists an open, dense subset $U$ of $X$ and a morphism

$$
\varphi: U \rightarrow G_{k}(N, n)
$$

such that for each $k$-point $x$ in $U, \varphi(x)$ corresponds to $T_{X, x}\left(T_{X, x}\right.$ denotes the tangent space of $X$ at $x$.)

Finally, we turn to the concept of generic subspace which will be used here. In the following all $U$ 's will denote non empty, open subsets of $\mathbf{P}_{k}^{N}$.

1) See for instance [1]: Let $p^{i_{1}}, \ldots, i_{N-n}$ be defined by $\left\{p_{i_{0}, \ldots, i_{n}}\right\}$ as in [1], Chapter VII. If the ideal $I(X)$ which defines $X$ in $\mathbf{P}_{k}^{N}=\operatorname{Proj}\left(k\left[X_{0}, \ldots, X_{N}\right]\right)$ is generated by $F_{1}, \ldots, F_{m}$, then $m \geq N-n$ and we may choose $U$ and number $F_{1}, \ldots, F_{m}$ such that the matrix

$$
\left(\begin{array}{l}
\frac{\partial F_{1}}{\partial X_{0}}(x), \ldots, \frac{\partial F_{1}}{\partial X_{N}}(x) \\
\frac{\partial F_{m}}{\partial X_{0}}(x), \ldots, \frac{\partial F_{m}}{\partial X_{N}}(x)
\end{array}\right)
$$

has the same rank as

$$
\left(\begin{array}{l}
\frac{\partial F_{1}}{\partial X_{0}}(x), \ldots, \frac{\partial F_{1}}{\partial X_{N}}(x) \\
\frac{\partial F_{N-n}}{\partial X_{0}}(x), \ldots, \frac{\partial F_{N-n}}{\partial X_{N}}(x)
\end{array}\right)
$$

Then the morphism $\varphi: U \rightarrow G_{k}(N, n)$ is given by

$$
\varphi(x)=\left\{p^{\left.i_{1}, \ldots, i_{N-n}\right\}}\right.
$$

where $p^{i_{1}, \ldots, i_{N-n}}$ is the minor obtained from the $i_{1}$ th., $\ldots, i_{N-n}$ th. columns of (2). 
Start with a $U_{1}$. For all $k$-points $\alpha_{1}$ in $U_{1}$ we give ourselves a $U_{2}\left(\alpha_{1}\right)$, for each $k$-point $\alpha_{2}$ in $U_{2}\left(\alpha_{1}\right)$ a $U_{3}\left(\alpha_{1}, \alpha_{2}\right)$, etc. We get the system

$$
\begin{aligned}
& U_{1} \\
& U_{2}\left(\alpha_{1}\right), \alpha_{1} \in U_{1} \\
& U_{3}\left(\alpha_{1}, \alpha_{2}\right), \alpha_{1} \in U_{1}, \alpha_{2} \in U_{2}\left(\alpha_{1}\right) \\
& \cdots \\
& U_{r}\left(\alpha_{1}, \ldots, \alpha_{r-1}\right), \alpha_{1} \in U_{1}, \ldots, \alpha_{r-1} \in U_{r-1}\left(\alpha_{1}, \ldots, \alpha_{r-2}\right)
\end{aligned}
$$

A system of non-empty open subsets as above will be denoted by $\mathscr{G}^{N-r}\left(\mathbf{P}_{k}^{N}\right), \mathscr{G}^{N-r}$ or $\mathscr{G}$ when no confusion is possible. Whenever the relation between $U$ 's and $\alpha$ 's are as above, and $\alpha_{r} \in U_{r}\left(\alpha_{1}, \ldots, \alpha_{r-1}\right)$, write

$$
\alpha=\left(\alpha_{1}, \ldots, \alpha_{r}\right) \in \mathscr{G}^{N-r} .
$$

Moreover, making some of the open subsets smaller, if necessary, one may assume that the subspace $P_{\alpha}$ defined by

$$
\begin{aligned}
& \alpha_{1,0} Z_{0}+\cdots+\alpha_{1, N} Z_{N}=0 \\
& \ldots \ldots \\
& \alpha_{r, 0} Z_{0}+\cdots+\alpha_{r, N} Z_{N}=0
\end{aligned}
$$

is of dimension $s=N-r$. This will always be assumed in the following.

Definition. A linear subspace $P^{s}$ of $\mathbf{P}_{k}^{N}$ is said to be a $\mathscr{G}^{s}$-space if $P^{s}=P_{\alpha}$ for some $\alpha \in \mathscr{G}^{s}$.

The following proposition follows easily from Bertinis first and second theorems:

Proposition 1.4. If $X$ is a non-singular subvariety of $\mathbf{P}_{k}^{N}$, of dimension $r \geq 2$. Then there exists $\mathscr{G}^{N-r+1}$ such that $X \cap P_{\alpha}$ is a nonsingular, reduced and irreducible curve for all $\alpha \in \mathscr{G}^{N-r+1}$.

Definition. A projective curve $X$ in $\mathbf{P}_{k}^{N}$ is said to be strange if 
it is not a $P^{1}$, and there exists a $k$-point $p$ of $\mathbf{P}_{k}^{N}$ such that $p \in T_{X, x}$ for all non-singular $k$-points $x$ of $X$.

The following theorem is due to E. Lluis (cf. [5] and [8]):

Theorem 1.5. If $k$ is of characteristic $\neq 2$, then there are no non-singular strange curves. In characteristic 2, the only non-singular strange curves are the plane conics.

§2. Birational unramified projections. The proof of the following proposition is straightforward:

Proposition 2.1. If $P \notin X$, then the morphism $\lambda_{P}: X \rightarrow Y$ is finite.

For all $y$ in $Y$, denote $\left(\left(\lambda_{P}\right)_{*}\left(\mathcal{O}_{X}\right)\right)_{y}$ by $\mathcal{O}_{X, y}$. Let $U=\operatorname{Spec}(\mathrm{A})$ be an open affine of $Y$ which contains the $k$-point $y$, and let $m_{y}$ be the maximal ideal of $y$. Then $\lambda_{p}^{-1}(U)=V=\operatorname{Spec}(B)$, where $B$ is a finite $A$-algebra (since $\lambda_{P}$ is finite).

Identify $A_{\mathrm{m}_{y}}$ with $\mathcal{O}_{Y, y}$ and $B_{\mathrm{m}_{y}}$ with $\mathcal{O}_{X, y}$ by the canonical isomorphisms. The semilocal ring $\mathcal{O}_{X, y}$ is finite over the local ring $\mathcal{O}_{Y, y}$ and $\lambda_{P}^{-1}(y)=\left\{x_{1} \ldots, x_{r}\right\}$ can be put in $1-1$ correspondence with the set $\left\{m_{1}, \ldots, m_{r}\right\}$ of maximal ideals in $\mathcal{O}_{X, y}$ in such a way that $\mathcal{O}_{X, x_{i}}=$ $\left(\mathcal{O}_{X, y}\right)_{\mathfrak{m} i}$. Let $n_{y}$ be the ideal in $\mathcal{O}_{X, y}$ generated by $\mathfrak{m}_{Y, y}$.

Recall that the affine tangent cone of $Y$ at $y$ is

$$
C_{Y, y}=\operatorname{Spec}\left(\operatorname{gr}_{\mathfrak{m}_{Y, y}}\left(\mathcal{O}_{Y, y}\right)\right) \text {. }
$$

Later $C_{Y, y}$ will be identified with a dense open subset of a projective scheme $\bar{C}_{Y, y}$ which is the closure of the union of all lines in $\mathbf{P}_{k}^{N-1}$ tangent to $Y$.

For $X$ we shall need the union of all lines tangent at some point of $\lambda_{P}^{-1}(y)$. Define

$$
C_{X, y}=\operatorname{Spec}\left(\operatorname{gr}_{n_{y}}\left(\mathcal{O}_{X, y}\right)\right)
$$

which is the union of all tangent cones of $X$ at the points of $\lambda_{P}^{-1}(y)$. There is a canonical morphism $c_{y}: C_{X, y} \rightarrow C_{Y, y}$. 
Now put $\mathcal{O}_{X, y}=R, \mathcal{O}_{Y, y}=\mathcal{O}, \mathrm{n}_{y}=\mathrm{n}, \mathrm{m}_{Y, y}=\mathrm{m}$ and $R_{\mathrm{m}_{i}}=\mathcal{O}_{i}, i=1, \ldots, r$. Since $R$ is finite over $\mathcal{O}$, the $\mathrm{n}$-adic completion $\hat{R}$ of $R$ is isomorphic to $R \otimes_{\mathcal{O}} \hat{\mathcal{O}}$, in particular the canonical $\varphi: \hat{\mathcal{O}} \rightarrow \hat{R}$ is injective. Moreover, as is easily seen, there is a canonical isomorphism

$$
\hat{R} \approx \Pi_{i=1}^{r} \hat{\mathcal{O}}_{i}
$$

such that the composition

$$
\hat{\mathcal{O}} \longrightarrow \Pi_{i=1}^{r} \hat{\mathcal{O}}_{i}
$$

is compatible with the canonical homorphisms

$$
\hat{\varphi}_{i}: \mathcal{O} \longrightarrow \hat{\mathcal{O}}_{i}
$$

The next proposition describes the action of a projection on $C_{X, y}$ and $C_{Y, y}$ :

Proposition 2.2. Assume that $X$ is non-singular and that $\lambda_{p}$ is unramified at all points of $\lambda_{p}^{-1}(y)$. Then $c_{y}$ is surjective, and each irreducible component $T_{i}$ of $C_{X, y}$ is mapped onto some irreducible component $C_{i}$ of $C_{Y, y}$. Moreover, $c_{y}$ induces an isomorphism

$$
c_{i}: T_{i} \approx C_{i} \text {. }
$$

Proof. $\varphi$ induces the graded homorphism $\psi: \operatorname{gr}_{\hat{\mathrm{m}}}(\hat{\mathcal{O}}) \rightarrow \operatorname{gr}_{\hat{\mathfrak{f}}}(\hat{R})$. It suffices to prove the claim for $c=\operatorname{Spec}(\psi)$ : Indeed, the following diagram commutes

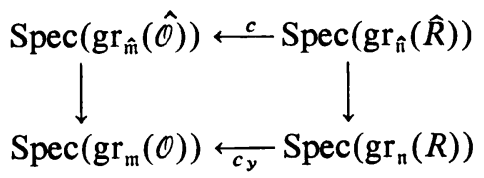

where the vertical arrows are the canonical isomorphisms.

Lemma 2.2.1. Let $\mathrm{n}_{i}=\operatorname{Ker}\left(\varphi_{i}\right)$. Then

i) $\mathrm{n}_{i}$ is a prime ideal, $i=1, \ldots, r$.

ii) $n_{1} \cap \ldots \cap n_{r}=(0)$ 
Let $\psi_{i}: \operatorname{gr}_{\hat{\mathrm{m}}}(\hat{\mathcal{O}}) \rightarrow \operatorname{gr}_{\mathrm{m}_{i}}\left(\hat{\mathcal{O}}_{i}\right)$ be the graded homomorphism induced by $\varphi_{i}$. Then

iii) $\psi_{i}$ is surjective

and if $\mathfrak{p}_{i}=\operatorname{Ker}\left(\psi_{i}\right)$.

iv) $\mathfrak{p}_{1} \cdots \cdot \mathfrak{p}_{r}=(0)$.

The lemma implies the propositions: Since $X$ is non-singular, $\operatorname{gr}_{\hat{t}_{i}}\left(\hat{O_{i}}\right)$ is isomorphic to a polynomial ring in $n=\operatorname{dim} X$ variables, so $\mathfrak{p}_{i}=\operatorname{Ker}\left(\psi_{i}\right)$ is a minimal prime. Thus by iv) $\operatorname{Spec}\left(\operatorname{gr}_{\hat{\mathrm{m}}}(\hat{\mathcal{O}})=V\left(\mathfrak{p}_{1}\right) \cup\right.$ $\ldots \cup V\left(\mathfrak{p}_{r}\right)$. On the other hand, the homomorphisms $\psi_{i}$ can be factored as follows:

$$
\operatorname{gr}_{\hat{\mathrm{m}}}(\hat{\mathcal{O}}) \longrightarrow \operatorname{gr}_{\hat{\mathrm{f}}}(\hat{R}) \longrightarrow \Pi_{i=1}^{r} \operatorname{gr}_{\hat{\mathrm{m}}_{\boldsymbol{i}}}\left(\hat{\mathcal{O}}_{i}\right) \longrightarrow \operatorname{gr}_{\hat{\mathrm{m}}_{i}}\left(\hat{\mathcal{O}}_{i}\right)
$$

and hence $\operatorname{Spec}\left(\psi_{i}\right)$ is the restriction of $\hat{c}$ to $T_{i}=\operatorname{Spec}\left(\operatorname{gr}_{\hat{A}_{i}}\left(\hat{\mathcal{O}}_{i}\right)\right), \quad i=$ $1, \ldots, r$. As $\psi_{i}$ is surjective, one thus gets an isomorphism of $T_{i}$ onto its image $V\left(\mathfrak{p}_{i}\right)$ in $\operatorname{Spec}\left(\operatorname{gr}_{\hat{\mathrm{m}}}(\hat{\mathcal{O}})\right)$.

Proof of the lemma: $\varphi_{i}$ is induced by the canonical unramified $f_{i}: \mathcal{O} \rightarrow \mathcal{O}_{i}$ which induces

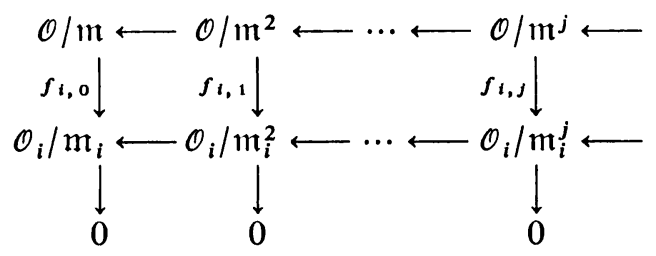

In fact, all is known except that $f_{i, j}$ is onto. But as $f_{i}$ is unramified, the restriction of $f_{i, j}$,

$$
f_{i, j}: \mathrm{m}^{j-1} / \mathrm{m}^{j} \longrightarrow \mathrm{m}_{i}^{j-1} / \mathrm{m}_{i}^{j}
$$

is onto for all $j$. Using this the claim follows by induction on $j$. Hence $\varphi_{i}$ and $\psi_{i}$ are onto. Further, since $\hat{\mathcal{O}}_{i}$ and $\hat{\mathcal{O}}$ have the 
same dimension, $\operatorname{Ker}\left(\varphi_{i}\right)$ are minimal primes of $\hat{\mathcal{O}}, i=1, \ldots, r$. Since the canonical $\mathcal{O C} \rightarrow \prod_{i=1}^{r} \hat{\mathcal{O}}_{i}$ sends $\alpha$ to $\left\{\varphi_{i}(\alpha)\right\}_{i=1, \cdots, r}$, one gets ii). For iv), note that

$$
\mathfrak{p}=\oplus_{j \geq 0}\left(n_{i} \cap m^{j}+\mathfrak{m}^{j+1} / \mathfrak{m}^{j+1}\right) .
$$

So $\quad\left(\mathfrak{p}_{1} \cdot \cdots \cdot \mathfrak{p}_{r}\right)_{j}=\left(\sum_{i_{1}+\cdots+i_{r}=j}\left(\prod_{v=1}^{r}\left(n_{v} \cap m^{i_{v}}+m^{i_{v}+1} / m^{i_{v}+1}\right)\right)\right) / m^{j+1}$. Thus as $\prod_{v=1}^{r}\left(n_{v} \cap m^{i_{v}}+\mathfrak{m}^{i_{v}+1}\right) \subseteq n_{1} \cdot \cdots \cdot n_{r}+\mathfrak{m}^{j+1},\left(\mathfrak{p}_{1} \cdot \cdots \cdot \mathfrak{p}_{r}\right)_{j}=(0)$, and the proof is complete.

Let $x$ be a $k$-point of $X$. With notation from $\S 1$, define

$$
\bar{C}_{X, x}=\pi_{x}\left(\ell_{x}^{-1}\left(\ell_{x}\left(\tilde{X}_{x}\right)\right)\right) \text { and } \bar{C}_{X,\left\{x_{1}, \ldots, x_{r}\right\}}=\cup_{i=1}^{r} \bar{C}_{X, x_{i}}
$$

for a set of $k$-points. When $\lambda_{P}^{-1}(y)=\left\{x_{1}, \ldots, x_{r}\right\}$, put $\bar{C}_{X, y}=\bar{C}_{X,\left\{x_{i}, \ldots, x_{r}\right\}}$.

Proposition 2.3. There is an open embedding $i_{x}: C_{X, x} \hookrightarrow \bar{C}_{X, x}$ which identifies $C_{X, x}$ with an open dense subset of $\bar{C}_{X, x}$.

Remark. If $x=(1: 0: \ldots: 0)$, then $i_{x}$ maps $C_{X, x}$ onto $D_{+}\left(Z_{0}\right) \cap$ $\bar{C}_{X, x}$, and is induced by the isomorphism $C_{\mathbf{P}_{k}^{N}, x} \stackrel{\approx}{\longrightarrow} D_{+}\left(Z_{0}\right)$, defined by the $k$-algebra isomorphism

$$
k\left[Z_{1} / Z_{0}, \ldots, Z_{N} / Z_{0}\right] \cong \operatorname{gr}_{\left(Z_{1} / Z_{0}, \ldots, Z_{N} / Z_{0}\right)}\left(k\left[Z_{1} / Z_{0}, \ldots, Z_{N} / Z_{0}\right]\right)
$$

which maps $Z_{i} / Z_{0}$ to $\left(Z_{i} / Z_{0} \bmod \left(Z_{1} / Z_{0}, \ldots, Z_{N} / Z_{0}\right)^{2}\right)$.

Proof of remark. ${ }^{2)}$ The blowing up diagram of $\S 1$ induces

$$
\begin{aligned}
& \widetilde{\mathbf{A}_{k}^{N}}=\pi_{x}^{-1}\left(D_{+}\left(Z_{0}\right)\right) \hookrightarrow \operatorname{Proj}\left(k\left[t_{1}, \ldots, t_{N}\right]\left[\bar{Z}_{1}, \ldots, \bar{Z}_{N}\right]\right) \\
& \downarrow \\
& \downarrow \\
& \mathbf{A}_{k}^{N}=\operatorname{Spec}\left(k\left[t_{1}, \ldots, t_{N}\right]\right)=D_{+}\left(Z_{0}\right) \quad \stackrel{\mathbf{P}}{N}_{k}^{N-1}=\operatorname{Proj}\left(k\left[\bar{Z}_{1}, \ldots, \bar{Z}_{N}\right]\right)
\end{aligned}
$$

where $t_{i}=Z_{i} / Z_{0} . \quad \widetilde{\mathbf{A}_{k}^{N}}$ is defined by the ideal

$$
\left(\left\{t_{i} \bar{Z}_{j}-t_{j} \bar{Z}_{i} \mid 1 \leq i, j \leq N\right\}\right) .
$$

hence $\widetilde{\mathbf{A}_{k}^{N}} \cap D_{+}\left(\bar{Z}_{i}\right)$ is the closed subscheme of $\operatorname{Spec}\left(k\left[t_{1}, \ldots, t_{N}, u_{1}, \ldots\right.\right.$, $\left.\left.u_{N}\right]\right), u_{j}=\bar{Z}_{j} \mid \bar{Z}_{i}$, defined by the ideal $\left(\left\{t_{i} u_{j}-t_{j} \mid 1 \leq j \leq N\right\}\right)$. Thus $\widetilde{\mathbf{A}_{k}^{N}} \cap$ $D_{+}\left(\bar{Z}_{i}\right)=\operatorname{Spec}\left(k\left[t_{i}, t_{1} / t_{i}, \ldots, t_{N} / t_{i}\right]\right)$, and moreover, the restriction $\pi_{x, i}$ of

2) This proof can be found in [6], p. 319 . 
$\pi_{x}$ is defined by the inclusion $k\left[t_{1}, \ldots, t_{N}\right] \hookrightarrow k\left[t_{i}, t_{1} / t_{i}, \ldots, t_{N} / t_{i}\right]$.

Note that the above proves the following lemma:

Lemma 2.4. The projection

$$
\bar{\zeta}_{(1: 0: \ldots: 0)}: D_{+}\left(Z_{0}\right)-\{(1: 0: \ldots: 0)\} \rightarrow \mathbf{P}_{k}^{N-1}
$$

maps the $k$-point $\left(1: \alpha_{1}: \ldots: \alpha_{N}\right)$ to $\left(\alpha_{1}: \ldots: \alpha_{N}\right)$.

Now let $\mathfrak{a}$ be the ideal in $k\left[t_{1}, \ldots, t_{N}\right]$ which defines the subscheme $X_{0}=X \cap D_{+}\left(Z_{0}\right)$ in $D_{+}\left(Z_{0}\right)=\operatorname{Spec}\left(k\left[t_{1}, \ldots, t_{N}\right]\right)$.

Then $\pi_{x, i}^{-1}\left(X_{0}\right)$ is defined by the ideal

$$
\mathfrak{a}_{i}=\mathfrak{a} k\left[t_{i}, t_{1} / t_{i}, \ldots, t_{N} / t_{i}\right] .
$$

Define

$$
\mathfrak{a}_{i}^{*}=\left\{F \in k\left[t_{i}, t_{1} / t_{i}, \ldots, t_{N} / t_{i}\right] \mid \exists m \text { such that } t_{i}^{m} F \in \mathfrak{a}_{i}\right\}
$$

Clearly $\mathfrak{a}_{i}^{*}$ is an ideal, and since $E=\pi_{x}^{-1}(x)$ is given in $\tilde{A}_{k}^{N} \cap D_{+}\left(\bar{Z}_{i}\right)=$ $\operatorname{Spec}\left(k\left[t_{i}, t_{1} / t_{i}, \ldots, t_{N} / t_{i}\right]\right)$ by the ideal $\left(t_{i}\right)$, it follows that

$$
\tilde{X}_{i}=\tilde{X} \cap\left(\tilde{\mathbf{A}}_{k}^{N} \cap D_{+}\left(\bar{Z}_{i}\right)\right) \hookrightarrow \operatorname{Spec}\left(k\left[t_{i}, t_{1} / t_{i}, \ldots, t_{N} / t_{i}\right]\right)
$$

is defined by the ideal $\mathfrak{a}_{i}^{*}$. Moreover,

$$
\tilde{X}_{i, x}=\tilde{X}_{x} \cap D_{+}\left(\bar{Z}_{i}\right)
$$

is defined by the ideal generated by $\mathfrak{a}_{i}^{*}$ and the image of $\left(t_{1}, \ldots, t_{N}\right)$, i.e. by the ideal

$$
\mathfrak{b}_{i}=\mathfrak{a}_{i}^{*}+\left(t_{i}\right)
$$

Hence $\tilde{X}_{i, x}$ is given as a subscheme of $E_{i}=\left[\tilde{\mathbf{A}}_{k}^{N} \cap D_{+}\left(\bar{Z}_{i}\right)\right]_{x}=\operatorname{Spec}\left(k\left[t_{i}\right.\right.$, $\left.\left.\left.t_{1} / t_{i}, \ldots, t_{N} / t_{i}\right] /\left(t_{i}\right)\right)=\operatorname{Speck}\left[t_{1} / t_{i}, \ldots, t_{N} / t_{i}\right]\right)$ by the ideal

$$
\widetilde{\mathfrak{b}}_{i}=\mathfrak{b}_{i} /\left(t_{i}\right)=\mathfrak{a}_{i}^{*}+\left(t_{i}\right) /\left(t_{i}\right) \text {. }
$$

To describe the ideal $\widetilde{\mathfrak{b}}_{i}$, we use the following notation: If

$$
F\left(t_{1}, \ldots, t_{N}\right)=F_{v_{1}}+\ldots+F_{v_{r}}
$$

where $F_{v_{1}}, \ldots, F_{v_{r}}$ are the homogeneous parts of $F$ of degrees $v_{1}<\ldots<v_{r}$, 
we put $\operatorname{in}(F)=F_{v_{1}}$.

It is then readily seen that

$$
\widetilde{\mathfrak{b}}_{i}=\left\{\varphi / t_{i}^{r} \mid \varphi=\operatorname{in}(F) \text { where } F \in \mathfrak{a} \text { and } v_{1}=r\right\} .
$$

Thus $\zeta_{x}\left(\tilde{X}_{i, x}\right)=D_{+}\left(\bar{Z}_{i}\right) \cap V_{+}\left(\operatorname{in}(\mathfrak{a})^{*}\right)$, where in $(\mathfrak{a})^{*}$ is the homogeneous ideal in $k\left[\bar{Z}_{1}, \ldots, \bar{Z}_{N}\right]$ obtained from in(a) by replacing $t_{j}$ by $\bar{Z}_{j}$. This gives

$$
\begin{aligned}
& \pi_{x}^{-1}\left(\zeta_{x}^{-1}\left(\ell_{x}\left(\tilde{X}_{i, x}\right)\right)\right)= \\
& \ell_{x}^{-1}\left(V_{+}\left(\operatorname{in}(\mathfrak{a})^{*}\right)\right) \cup\{x\}=V(\operatorname{in}(\mathfrak{a}))
\end{aligned}
$$

by means of Lemma 2.4. This completes the proof of the proposition.

Corollary 2.5. If $x$ is a non-singular $k$-point of $X$, then $\bar{C}_{X, x}=$ $T_{X, x}$

Proof. Immediate from the remark following Proposition 2.3.

The following proposition describes how a projection acts on the tangent space:

Proposition 2.6. Assume $P \notin X$, and that $\lambda_{p}$ is unramified. Let $y \in Y$ be a k-point. Then $\lambda_{p}$ induces a morphism $\varphi_{p}: \bar{C}_{X, y} \rightarrow \bar{C}_{Y, y}$ which makes the following diagram commutative:

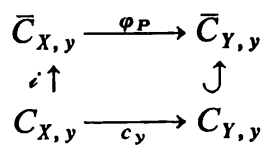

Remark. For each $x_{i} \in \lambda_{P}^{-1}(y)$ there is an open embedding $i_{i}$ : $C_{X, x_{i}} \hookrightarrow \bar{C}_{X, x_{i}}$. Since $C_{X, y}$ is the disjoint union of all $C_{X, x_{i}}$ 's, and by definition $\bar{C}_{X, x}=\cup_{i=1}^{r} \bar{C}_{X, x_{i}}, i_{1}, \ldots, i_{r}$ induce a morphism $\because: C_{X, y} \rightarrow \bar{C}_{X, y}$.

Proof of the proposition. Enough to show that the following commutes

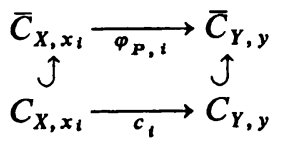


where $c_{i}$ is induced by the canonical $f_{i}: \mathcal{O}_{Y, y} \rightarrow \mathcal{O}_{X, x_{i}}$. One may assume $x_{i}=$ $(1: 0: 0: \ldots: 0), P=(0: \ldots: 0: 1)$ and hence $y=(1: 0: \ldots: 0)$. Put $Z_{i} / Z_{0}=$ $t_{i}, \bar{Z}_{i} / \bar{Z}_{0}=\bar{t}_{i}$. Then the projection $\swarrow_{P}: D_{+}\left(Z_{0}\right) \rightarrow D_{+}\left(\bar{Z}_{0}\right)$ is given by the homomorphism $k\left[\bar{t}_{1}, \ldots, \bar{t}_{N-1}\right] \hookrightarrow k\left[t_{1}, \ldots, t_{N}\right]$ which maps $\bar{t}_{i}$ to $t_{i}$. The maximal ideals which correspond to $x_{i}$ and $y$ are $m=\left(t_{1}, \ldots, t_{N}\right)$ and $\mathfrak{n}=\left(\bar{t}_{1}, \ldots, \bar{t}_{N-1}\right)$, respectively. Let $\mathfrak{a}$ and $\mathfrak{b}$ be the ideals in $k\left[t_{1}\right.$, $\left.\ldots, t_{N}\right]$ and $k\left[\bar{t}_{1}, \ldots, \bar{t}_{N-1}\right]$ which define $X \cap D_{+}\left(X_{0}\right)$ and $Y \cap D_{+}\left(Z_{0}\right)$. Finally $u_{1}, \ldots, u_{N}$ and $\bar{u}_{1}, \ldots, \bar{u}_{N-1}$ denote the images of the $t$ 's and ${ }^{\prime}$ 's in $m / \mathfrak{m}^{2}$ and $n / \mathfrak{n}^{2}$, respectively. The following diagram commutes:

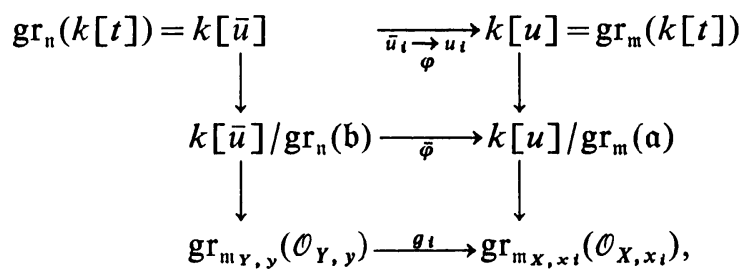

where the ideal $\oplus_{i \geq 0} \mathfrak{a} \cap \mathrm{m}^{i}+\mathrm{m}^{i+1} / \mathrm{m}^{i+1}$ in $\operatorname{gr}_{\mathfrak{m}}(k[t])$ is denoted by $\operatorname{gr}_{\mathfrak{m}}(\mathfrak{a})$. If $\bar{m}$ is the image of $m$ in $k[t] / \mathfrak{a}$, it is readily seen that we get an exact sequence

$$
0 \longrightarrow \mathrm{gr}_{\mathrm{m}}(\mathfrak{a}) \longrightarrow \mathrm{gr}_{\mathrm{m}}(k[t]) \longrightarrow \mathrm{gr}_{\mathrm{m}}(k[t] / \mathfrak{a}) \longrightarrow 0 .
$$

Furthermore, $\bar{\varphi}$ is the canonical homomorphism induced by $\varphi$, and $g_{i}$ the one induced by $f_{i}$.

Since $\quad c_{i}=\operatorname{Spec}\left(g_{i}\right)$ and $\operatorname{Spec}(\bar{\varphi})=\ell_{P}^{\prime}: \bar{C}_{X, x_{i}} \cap D_{+}\left(Z_{0}\right) \rightarrow \bar{C}_{Y, y} \cap D_{+}\left(\bar{Z}_{0}\right)$ is induced by $\overline{\zeta_{P}}$, the proof is complete.

Under the assumptions in Proposition 2.6, $\lambda_{P}$ induces a morphism $c: C_{X, y} \rightarrow C_{Y, y}, y=\lambda_{P}(x)$, which is onto and maps each irreducible component $T_{1}, \ldots, T_{r}\left(\lambda_{P}^{-1}(y)=\left\{x_{1}, \ldots, x_{r}\right\}\right)$ of $C_{X, y}$ onto an irreducible component $C_{i}$ of $C_{Y, y}$, inducing an isomorphism $\bar{c}_{i}: T_{X, x_{i}} \rightarrow C_{i}$. It follows that $\bar{C}_{Y, y}$ is a union of $P^{n}$ 's in $\mathbf{P}_{k}^{N-1}$. In general the number of irreducible components of $C_{Y, y}$ and $\bar{C}_{Y, y}$ is less than or equal to $r$, the number of points in $\lambda_{P}^{-1}(y) . \quad\left(r=e\left(\mathcal{O}_{Y, y}\right)\right.$, the multiplicity, cf. [9], Vol II, Ch. VIII Corollary 1 of Theorem 24.)

Definition. We say that $y$ is an ordinary singularity if $C_{Y, y}$ has $r$ irreducible components. 


\section{Chapter II. Projections to $\mathbf{P}_{k}^{2 n}$}

§1. Embedded, projective varieties with a tangent condition. The aim of this paragraph is to study embedded, projective varieties $X \hookrightarrow$ $\mathbf{P}_{\boldsymbol{k}}^{N}$ which, loosely speaking, have the property that for any two regular point: $x, y$ of $X$, the tangent spaces $T_{X, x}$ and $T_{X, y}$ are very close to each other. More precisely, we prove and deduce some consequences of the following:

Theorem 1.1. Let $X$ be a closed, n-dimensional subvariety of $\mathbf{P}_{k}^{N}$. Assume that for any two regular k-points $x$ and $y$ in $X$, the linear subspace of $\mathbf{P}_{k}^{N}$ spanned by $T_{X, x}$ and $T_{X, y}$, which we denote by $<T_{X, x}$, $T_{X, Y}>$ is of dimension $\leq n+1$. Then either

i) There exists an n+1-dimensional linear subspace $P^{n+1}$ of $\mathbf{P}_{k}^{N}$, such that $X \subset P^{n+1}$,

or

ii) There exists an $n-1$-dimensional linear subspace $L^{n-1}$ of $\mathbf{P}_{k}^{N}$, such that for all regular k-points $x \in X, T_{X, x} \supset L^{n-1}$.

Proof. Let $U$ be the open set of Proposition 1.3 in Chapter I. If $T_{X, x}=T_{X, y}$ for all $k$-points $x$ and $y$ in $U$, the claim is trivial: Indeed, it then follows that $U \subset T_{X, x_{0}}$ for a fixed point $x_{0}$, hence $X \subset T_{X, x_{0}}$ i.e. $X=T_{X, x_{0}}$. So we may assume that there are $k$-points $x_{1}, x_{2} \in U$ such that $<T_{X, x_{1}}, T_{X, x_{2}}>=P^{n+1}$. Let $T_{X, x_{1}} \cap T_{X, x_{2}}=L^{n-1}$. We show that $P^{n+1}$ and $L^{n-1}$ satisfy the claim of the proposition.

Lemma 1.2. $\left\{x \in U(k) \mid T_{X, x} \supset L\right\}=\Delta(U, L)(k)$, where $\Delta(U, L)$ is a closed subset of $U$.

Proof. Let $\varphi$ be the morphism of proposition 1.3 in Chapter I. With notation from there, we have

$$
\Delta(U, L)=\varphi^{-1}(\Delta(L)) .
$$

If now $\Delta(U, L)=U$, then ii) holds. If not, then $U-\Delta(U, L)=V$ is an open, dense subset of $X$. To prove i), it suffices to show that $V \subset P^{n+1}$. 
So let $x \in V$. Put $L_{i}=T_{X, x_{i}} \cap T_{X, x} i=1,2$. Then $L, L_{1}$ and $L_{2}$ are distinct $n-1$-dimensional subspaces of $\mathbf{P}_{k}^{N}$ : In fact, they are $n-1$ dimensional since the intersection of two distinct hyperplanes in some $\mathbf{P}_{k}^{m}$ is $m$-2-dimensional, so since $\left\langle T_{X, x_{i}}, T_{X, x}\right\rangle$ is $n+1$-dimensional as $x \in V, L_{i}$ is $n$-1-dimensional. Further, $L_{i} \neq L$ since $L_{i}=L$ implies $T_{X, x} \supset L . \quad L_{1} \neq L_{2}$, since $L_{1}=L_{2}$ means that $T_{X, x_{1}} \cap T_{X, x}=T_{X, x_{2}} \cap T_{X, x}$, so $\operatorname{L} \cap T_{X, x}=T_{X, x_{1}} \cap T_{X, x_{2}} \cap T_{X, x}=L_{1}$. Thus $L_{1} \subseteq L$, i.e. $L_{1}=L$, which contradicts what we have found above.

Since $L_{1}, L_{2}$ and $L$ are distinct $n+1$-dimensional subspaces,

$$
\begin{aligned}
& <L, L_{i}>=T_{X, x_{i}}, \quad i=1,2 \\
& <L_{1}, L_{2}>=T_{X, x}
\end{aligned}
$$

Hence

$$
\begin{aligned}
& <L, L_{1}, L_{2}>=<T_{X, x_{1}}, T_{X, x_{2}}, T_{X, x}>= \\
& <<L_{1}, L>, \quad<L, L_{2}>>=<T_{X, x_{1}}, T_{X, x_{2}}>=P^{n+1}
\end{aligned}
$$

Thus $x \in P^{n+1}$ and the claim follows. This completes the proof of Theorem 1.1.

In the statements of Theorem 1.1, i) tells that $X$ is contained in a low dimensional subspace of $\mathbf{P}_{k}^{N}$. In general, ii) need not imply this. However, if $X$ is non-singular, then the following holds:

Theorem 1.3. Let $X$ be a non-singular, closed subvariety of $\mathbf{P}_{k}^{N}$, of dimension $n$. Assume that ii) in Theorem 1.1 holds, and that $N \geq 2 n+1$. Then $X$ is contained in some hyperplane $H=P^{N-1}$ of $\mathbf{P}_{k}^{N}$.

Proof. We need the following result (cf. [4], Corollary 2 of Lemma 1.):

Lemma 1.4. ${ }^{3)}$ Let $X$ be an n-dimensional subvariety of $\mathbf{P}_{k}^{N}$.

3) The following neat proof of this Lemma was pointed out by the referee: Let $L$ be the linear system of hyperplane-sections of $X$. Then $X \nsubseteq$ Hyperplane $\Leftrightarrow$ $\operatorname{dim} L=N$.

If $D=H \cdot X, H$ a hyperplane, is a general member of $L$, then $\operatorname{dim} \operatorname{tr}_{D} L=\operatorname{dim} L-1$ So $X \Phi$ Hyperplane $\Leftrightarrow D \Phi$ Hyperplane, and the proof can be completed by induction, 
Let $t$ be a positive integer such that $n+t \geqq N$. Suppose that for a generic $P^{t}, P^{t} \cap X$ is contained in some $t-1$-dimensional subspace $Q^{t-1}$ of $\mathbf{P}_{k}^{N}$. Then $X$ is contained in a hyperplane of $\mathbf{P}_{k}^{N}$.

Put $t=N-n+1$. Then $t \geq 2 n+1-n+1>2$, so in order to prove Theorem 1.3, it suffices to show

Lemma 1.5. Let the situation be as in Theorem 1.3. Then there exists $\mathscr{G}^{t}$ such that for all $\mathscr{G}^{t}$-spaces $P^{t}, P^{t} \cap X$ is a plane curve.

Proof. By Proposition 1.4, Chapter I there exists $\mathscr{G}^{t}$ such that all $\mathscr{G}^{t}$-spaces $P_{\alpha}^{t}$ intersect $L$ in a point $p_{\alpha}$ and intersect $X$ in a nonsingular irreducible curve $X_{\alpha}$. Now $X_{\alpha}$ has the property that all tangents meet in $p_{\alpha}$ : In fact, let $x$ be a $k$-point of $X_{\alpha}$ different from $p_{\alpha}$. Then the line $T_{X_{\alpha}, x}$ and the hyperplane $L$ of $T_{X, x}=P^{n}$ meet in a point, say $p^{\prime}$ : On the other hand, $T_{X, x} \subset P_{\alpha}^{t}$, so $p^{\prime} \in L \cap P_{\alpha}^{t}=\left\{p_{\alpha}\right\}$. Now the proof of Lemma 1.5, and hence of Theorem 1.3 is completed by Theorem 1.5 of Chapter I.

§2. The strange subset of $\mathbf{X} \times{ }_{k} \mathbf{X}$. The following notion is motivated by the tangent condition of the previous paragraph.

Proposition 2.2. There exists a closed subset $\operatorname{St}(X)$ of $X \times{ }_{k} X$, such that $\operatorname{St}(X)(k)=\left\{(x, y) \mid \operatorname{dim}<T_{X, x}, T_{X, y}>\leq n+1\right\}$.

Proof. Suppose $X=\operatorname{Proj}\left(k\left[X_{0}, \ldots, X_{N}\right] /\left(F_{1}, \ldots, F_{m}\right)\right)$. The subspace $T_{X, x}$ of $\mathbf{P}_{k}^{N}$ is given by

$$
\sum_{i=0}^{N} X_{i} \frac{\partial F_{\alpha}}{\partial X_{i}}(x)=0, \quad \alpha=1, \ldots, m .
$$

Let $T_{X, x}^{*}$ denote the affine cone over $T_{X, x}$, i.e. the linear subspace of $\mathbf{A}_{k}^{N+1}$ which is the closure of the inverse image of $T_{X, x}$ under the canonical morphism

$$
\mathbf{A}_{k}^{N+1}-\{(0, \ldots, 0)\} \longrightarrow P_{k}^{N} .
$$

If $\mathbf{A}_{k}^{N+1}=\operatorname{Spec}\left(k\left[X_{0}, \ldots, X_{N}\right]\right)$, then $T_{X, x}^{*}$ is the subspace defined by 
(2.2.1). With this notation, the condition

$$
\operatorname{dim}<T_{X, x}, T_{X, y}>\leq n+1
$$

is equivalent to

$$
\operatorname{dim}<T_{X, x}^{*}, T_{X, y}^{*}>\leq n+2
$$

which in turn is equivalent to

$$
\operatorname{dim} T_{X, x}^{*} \cap T_{X, y}^{*} \geq 2(n+1)-(n+2)=\mathrm{n} .
$$

Now $T_{X, x}^{*} \cap T_{X, y}^{*} \subset \mathbf{A}_{k}^{N+1}$ is defined by

$$
\sum_{i=0}^{N} X_{i} \frac{\partial F_{\alpha}}{\partial X_{i}}(x)=0, \quad \alpha=1, \ldots, m
$$

$$
\sum_{i=0}^{N} X_{i} \frac{\partial F_{\alpha}}{\partial X_{i}}(y)=0, \quad \alpha=1, \ldots, m
$$

and thus, finally, the condition is equivalent to

$$
\operatorname{rk}\left[\begin{array}{l}
\frac{\partial\left(F_{1}, \ldots, F_{m}\right)}{\partial\left(X_{0}, \ldots, X_{N}\right)}(x) \\
\frac{\partial\left(F_{1}, \ldots, F_{m}\right)}{\partial\left(X_{0}, \ldots, X_{N}\right)}(y)
\end{array}\right] \leqq N+1-n
$$

Now let $F$ be the closed subset of

$$
X^{*} \times{ }_{k} X^{*}=\operatorname{Spec}\left(S \otimes_{k} S\right)=\operatorname{Spec}(k[X, Y] / J)
$$

where

$$
J=\left(F_{1}(X), \ldots, F_{m}(X), F_{1}(Y), \ldots, F_{m}(Y)\right)
$$

defined by the ideal generated by $J$ and all $N+1-n$-minors of the matrix

$$
\left[\begin{array}{l}
\frac{\partial\left(F_{1}, \ldots, F_{m}\right)}{\partial\left(X_{0}, \ldots, X_{N}\right)}\left(X_{0}, \ldots, X_{N}\right) \\
\frac{\partial\left(F_{1}, \ldots, F_{M}\right)}{\left.\partial\left(X_{0}, \ldots, X_{N}\right)\right)}\left(Y_{0}, \ldots, Y_{N}\right)
\end{array}\right]
$$

We then get $\operatorname{St}(X)$ as the image of $F \cap U$ by the canonical $\psi: U=\left(X^{*}-\{(0, \ldots, 0)\}\right) \times{ }_{k}\left(X^{*}-\{(0, \ldots, 0)\} \rightarrow X \times{ }_{k} X\right.$. 
Since the polynomials which define $F$ are homogeneous in the variables $X_{0}, \ldots, X_{N}$ and in the variables $Y_{0}, \ldots, Y_{N}$, this is a closed subset of $X \times{ }_{k} X$.

§3. Projections of algebraic varieties to $\mathbf{P}_{\boldsymbol{k}}^{2 n}$. We are going to prove the following:

Theorem 3.1. Let $X$ be a projective, non-singular variety, defined over the algebraically closed field $k$. Assume that $X$ is embedded as a closed subvariety of $\mathbf{P}_{k}^{2 n+1}$ where $n=\operatorname{dim}(X)$.

Then there exists a point $P \in \mathbf{P}_{k}^{2 n+1}$ such that the projection $\ell_{P}$ induces a morphism

$$
\lambda: X \longrightarrow Y
$$

where $Y$ is a closed subvariety of $\mathbf{P}_{k}^{2 n} . \lambda$ is an isomorphism except at a finite number of $k$-points $y_{1}, \ldots, y_{s}$ in $Y$, which are ordinary double points of $Y$.

We need the following result from [2] (Corollary 3.3):

Proposition 3.2. Let $X=\operatorname{Proj}\left(k\left[\xi_{0}, \ldots, \xi_{2 n+1}\right]\right)$ be a closed subvariety of $P_{k}^{2 n+1}$, which is non-singular of dimension $n$. Let

$$
F\left(X_{i j} \mid 0 \leq i \leq 2 n, 0 \leq j \leq 2 n+1\right)
$$

be a non-zero polynomial with coefficients from $k$.

Then there exist linear combinations in $\xi_{0}, \ldots, \xi_{2 n+1}$ with coefficients from $k$,

$$
\zeta_{i}=\alpha_{i, 0} \xi_{0}+\cdots+\alpha_{i, 2 n+1} \xi_{2 n+1}, \quad i=1, \ldots, 2 n
$$

such that $F\left(\alpha_{i, j}\right) \neq 0$ and such that if $T=k[\zeta]$, then the inclusion $T \hookrightarrow S=k[\xi]$ induces a morphism

$$
\lambda: X \rightarrow Y=\operatorname{Proj}(T)
$$

which is unramified and an isomorphism except at a finite number of $k$-points, which are double points of $Y$. 
Clearly the conclusion of this proposition may be rephrased as follows: For any non-empty open subset $U$ of $\mathbf{P}_{k}^{2 n+1}$, there exists $P \in U$ such that the projection $\ell_{P}$ has the property of the theorem, except possibly for $y_{1}, \ldots, y_{s}$ being ordinary singularities.

The proof of Theorem 3.1 now amounts to finding a non-empty open subset $U$ of $\mathbf{P}_{k}^{2 n+1}$ such that if $P \in U$ satisfies Proposition 3.2, then $Y=\ell_{P}(X)$ has at most ordinary singularities.

First, if $\operatorname{St}(X)=X \times{ }_{k} X$ then by Theorem 1.3, $X$ is contained in a hyperplane of $\mathbf{P}_{k}^{2 n+1}$, so the claim is trivial. If on the other hand $\operatorname{St}(X) \neq X \times{ }_{k} X$, then by Proposition 2.2

$$
\operatorname{dim}\left(\operatorname{St}(X)-\Delta_{X / k}\right) \leqq 2 n-1
$$

Hence by Chapter I, Corollary 1.2

$$
\operatorname{dim} L\left(\operatorname{St}(X)-\Delta_{X / k}\right) \leq 2 n
$$

Therefore $U=\mathbf{P}_{k}^{2 n+1}-L\left(S t(X)-\Delta_{X / k}\right)$ is a non-empty open subset.

Suppose now that $P \in U$ satisfies the conclusion of Proposition 3.2. To show is that for all $y \in B\left(\lambda_{P}\right)$

$$
C_{Y, y}=\operatorname{Spec}\left(\operatorname{gr}_{m_{Y, y}}\left(\mathcal{O}_{Y, y}\right)\right)
$$

has exactly two irreducible components, cf. page 315 .

Since $y$ is a double point and $\lambda_{P}: X \rightarrow Y$ is unramified, $\lambda_{P}^{-1}(y)=$ $\left\{x_{1}, x_{2}\right\}$, see page 315 .

The projection $\ell_{P}$ maps $T_{X, x_{1}}$ and $T_{X, x_{2}}$ onto distinct $n$-dimensional subspaces of $\mathbf{P}_{k}^{2 n}$. In fact, if $\ell_{P}\left(T_{X, x_{1}}\right)=\ell_{P}\left(T_{X, x_{2}}\right)$, then

$$
<T_{X, x_{1}} T_{X, x_{2}}>=<T_{X, x_{1}}, P>
$$

is an $n+1$-dimensional subspace, contradicting $P \notin L\left(\operatorname{St}(X)-\Delta_{X / k}\right)$.

By Proposition 2.3, Corollary 2.5 and Proposition 2.6 of Chapter I, it now follows that

$$
c_{y}: C_{X,\left\{x_{1}, x_{2}\right\}} \rightarrow C_{Y, y}
$$

maps the two irreducible components of $C_{Y, y}$ onto distinct irreducible components of $C_{Y, y}$. Thus the claim follows by Chapter I, Proposition 2.2. 
Tufts University, Medford, Mass. U.S.A. University of Bergen, Bergen, Norway.

\section{References}

EGA Grothendieck, A.: “Elements de géometrie algébrique”, Publ. Math. de l'Institut des Hautes Études Scientifiques.

[1] Hodge, W. V. D and Pedoe, D: "Methods in algebraic geometry", Volume I, Cambridge University Press 1968.

[2] Holme, A.: "Formal embedding and projection theorems" American Journal of Mathematics 1971.

[3] Lluis, E.: "Sur l'immersion des variétés algébriques" Annals of Mathematics, 1955.

[4] Lluis, E.: "De las singularidades que aparecen al proyectar variedades algebraicas" Publ. Mat. de Soc. Mat. Mexicana 1956.

[5] Lluis, E.: "Variedades algebraicas con ciertas condiciones en sus tangentes" Publ. Mat. de Soc. Mat. Mexicana 1962.

[6] Mumford, D.: “Introduction to algebraic geometry". Lecture notes, Harvard University 1966.

[7] Roberts, J.: "Generic projections of algebraic varieties". American Journal of Mathematics 1971.

[8] Samuel, P.: "Old and new results on algebraic curves". Tata Institute 1966.

[9] Zariski and Samuel: "Commutative Algebra", Vol. I and II, D. Van Nostrand, Princeton, 1958. 